\title{
Highly efficient grating coupler between optical fiber and silicon photonic circuit
}

\author{
Shankar Kumar Selvaraja, Diedrik Vermeulen, Marc Schaekers ${ }^{*}$, Erik Sleeckx ${ }^{*}$, \\ Wim Bogaerts, Gunther Roelkens, Pieter Dumon, Dries Van Thourhout, Roel Baets \\ Gent University-IMEC , Department of Information Technology, Gent, Belgium, \\ IMEC, Leuven, Belgium \\ shankar@intec.ugent.be
}

\begin{abstract}
: we report on a highly efficient grating coupler between an optical fiber and a silicon photonic circuit. Using layers of $\mathrm{Si} / \mathrm{SiO} 2$ as a Bragg mirror and amorphous Si we have measured a coupling efficiency of $69.5 \%$.

(2009 Optical Society of America

OCIS codes: (230.3120) Integrated optics Devices; (230.1950) Diffraction gratings; (250.5300) Photonic integrated circuits; (230.7370) Waveguides.
\end{abstract}

\section{Introduction}

Due to the high refractive index contrast, Silicon-on-Insulator (SOI) technology is an attractive platform for compact integrated photonic circuits. SOI is also CMOS-compatible and has created widespread interest not only in the photonics community, but also in the electronics world to integrate photonic functions in the next generation of high speed processors. However, efficient coupling in and out of a silicon circuit remains a challenge, due to a huge mismatch between the optical fiber mode and the nanophotonic waveguide mode.

Several attempts were made to find a solution to this problem [1,2]. While inverted taper couplers with overlay provide large bandwidth and efficiency, often they require post processing and can not be used for wafer scale testing [2, 3]. Grating couplers are an attractive solution for wafer scale testing, since light can be coupled out of plane [1]. To increase the efficiency, the light radiated towards the substrate can be reflected upwards by using a mirror. This mirror can be a metal layer or a Bragg mirror. Using gold as bottom mirror an efficiency of $69 \%$ was demonstrated for a 10 by $10 \mu \mathrm{m}$ grating coupler [4]. This process involves bonding and substrate removal, which is complex and expensive, and since gold is being used, this process is not CMOS-compatible.

In this paper, we reflect the downwards radiating light by using a stack of amorphous-Si/ $\mathrm{SiO}_{2}$ Bragg bottom reflectors. The waveguides were fabricated on a $200 \mathrm{~mm}$ Si wafer with CMOS-compatible fabrication processes. The temperature of each fabrication step was kept under $450^{\circ} \mathrm{C}$ to make the process CMOS back-end compatible. We demonstrate a coupling efficiency of $69.5 \%$ between an optical fiber and a SOI waveguide.

\section{Design}

We optimized the coupling structure with a particle swarm optimization algorithm for a silicon waveguide core thickness of 220nm. The field profile of the optimal design is shown in Figure 1.
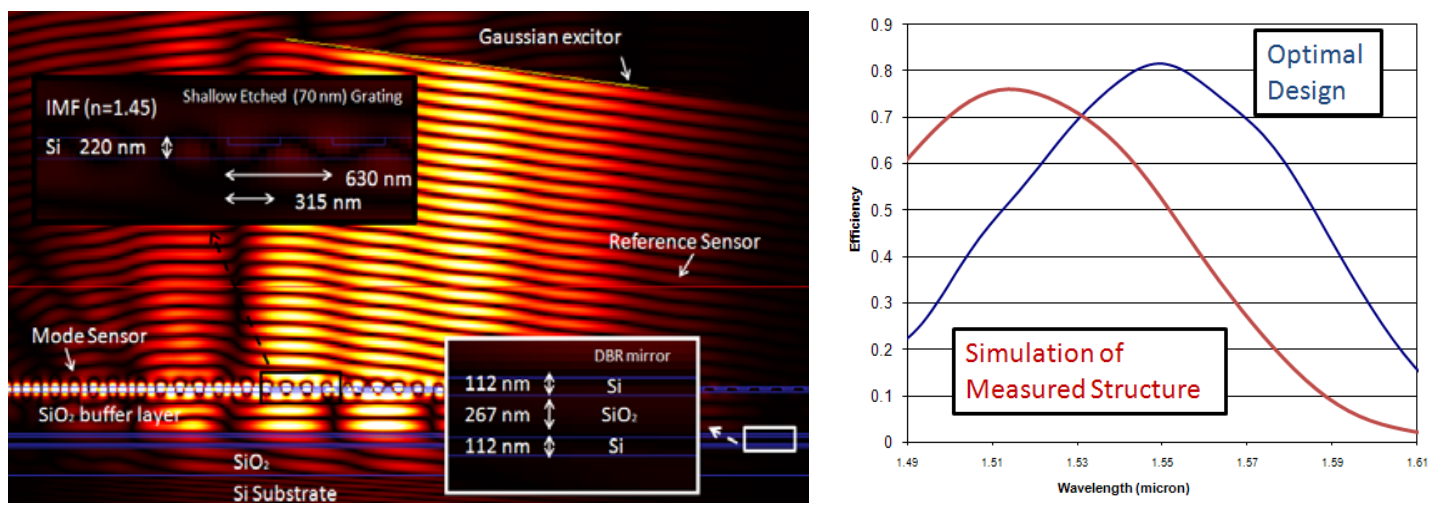

Fig. 1 (left) Simulated field profile of grating coupler with Bragg bottom mirror. (right) Simulated coupling efficiency. 
The period of the grating is $630 \mathrm{~nm}$ with a duty cycle of $50 \%$ and the etch depth is $70 \mathrm{~nm}$. The number of grating periods is 25. If the thickness of the $\mathrm{SiO}_{2}$ buffer layer is $1.44 \mu \mathrm{m}$, the Bragg mirror will reflect the light constructively. The fiber is tilted under an angle of 10 degrees to avoid a large second order reflection. For this angle the optimal waveguide width of the grating is $15 \mu \mathrm{m}$. The refractive index of the surrounding medium is 1.45 , which corresponds to $\mathrm{SiO}_{2}$ or Index Matching Fluid (IMF). Theoretically the coupling efficiency is $82 \%$ for TE polarized light at 1550nm (see Figure 1).

\section{Fabrication and characterization}

The grating fiber couplers with bottom mirrors were fabricated on a $200 \mathrm{~mm} \mathrm{Si}$ wafer. First a $1 \mu \mathrm{m} \mathrm{SiO}_{2}$ isolation layer is deposited on a bare Si wafer. Then the Bragg mirror, consisting of two 112nm layers of a-Si and a $267 \mathrm{~nm}$

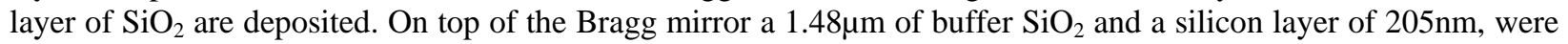
deposited [5]. The grating couplers and waveguides were fabricated using 193nm optical lithography and dry etching [6].

The coupling efficiency is characterized with a fiber-to-fiber measurement. The test structures consist of identical input and output gratings couplers connected by a waveguide. Fiber-to-fiber transmission is measured by launching light into the input coupler from a single mode fiber connected to a broadband light source (SLED) and the light from the output coupler is captured by another single mode fiber connected to a spectrum analyzer. Both the fibers were positioned at an angle of 10 degrees to the sample normal and index matching fluid was applied between the optical fiber facet and the fiber coupler to avoid reflections at the fiber facets. The measured coupling efficiency is $69.5 \%$ for a single grating coupler with a $1 \mathrm{~dB}$-bandwidth of $36 \mathrm{~nm}$ and a $3 \mathrm{~dB}$-bandwidth of $63 \mathrm{~nm}$ (Fig. 2 ). The simulated efficiency for the fabricated device is $76 \%$ as shown in Figure 1 . The non optimal waveguide width of $10 \mu \mathrm{m}$ explains $5 \%$ of the difference in coupling efficiency between measurement and simulation.
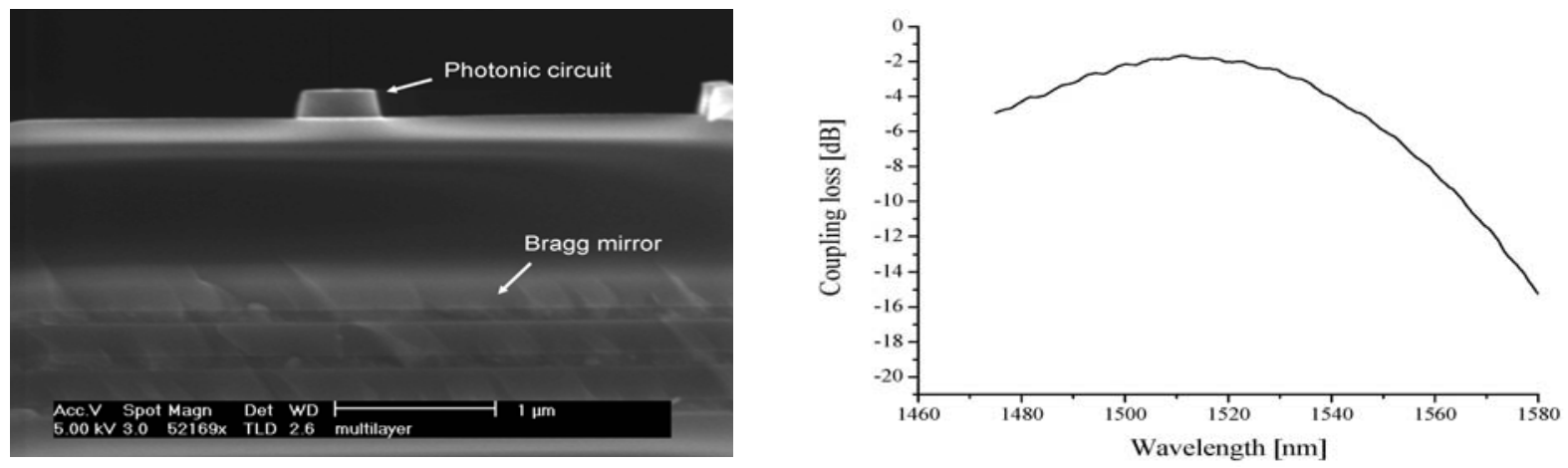

Fig.2 (left) Cross-section image of the fabricated structure. (right) Measured coupling efficiency of a grating coupler with Bragg bottom mirror.

\section{Conclusion}

We have demonstrated a highly efficient grating coupler between optical fiber and silicon waveguide. Experimentally we have measured a coupling efficiency of $69.5 \%$ for grating couplers with Bragg bottom mirror. High efficiency, low temperature and, wafer scale approach demonstrates its potential for high volume telecommunication and integrated electronic-photonics applications.

\section{Reference}

[1] D. Taillaert, F. Van Laere, M. Ayre, W. Bogaerts, D. Van Thourhout, P. Bienstman, and R. Baets, "Grating couplers for coupling between optical fibers and nanophotonic waveguides," JJAP Part-1 45, 6071-6077 (2006.)

[2] G. Roelkens, P. Dumon, W. Bogaerts, D. Van Thourhout, and R. Baets, "Efficient silicon-on-insulator fiber coupler fabricated using 248-nm-deep UV lithography," IEEE PTL, 17, 2613-2615 (2005).

[3] T. Tsuchizawa, K. Yamada, H. Fukuda, T. Watanabe, J. Takahashi, M. Takahashi, T. Shoji, E. Tamechika, S. Itabashi, and H. Morita, "Microphotonics devices based on silicon microfabrication technology," IEEE JSTQE, 11, 232-240 (2005).

[4] F. Van Laere, G. Roelkens, M. Ayre, J. Schrauwen, D. Taillaert, D. Van Thourhout, T. E. Krauss, and R. Baets, "Compact and highly efficient grating couplers between optical fiber and nanophotonic waveguides," JLT, 25, 151-156 (2007).

[5] S. K. Selvaraja, E. Sleeckx, W. Bogaerts, M. Schaekers, P. Dumon, D. Van Thourhout, and R. Baets, "Low loss amorphous silicon photonic wire and ring resonator fabricated by CMOS process," in European Conference on Optical Communication, Germany, 2007, PD 2.2 .

[6] S. K. Selvaraja, P. Jaenen, S. Beckx, W. Bogaert, P. Dumon, D. Van Thourout, and R. Baets, "Silicon nanophotonic wire structures fabricated by 193nm optical lithography," in Lasers and Electro-Optics Society, 2007 (The 20th Annual Meeting of the IEEE LEOS, Florida, 2007), pp. 48-49. 